PROCEEDINGS OF THE

AMERICAN MATHEMATICAL SOCIETY

Volume 136, Number 7, July 2008, Pages 2571-2573

S 0002-9939(08)09148-X

Article electronically published on February 29, 2008

\title{
A FORMULA FOR THE EULER CHARACTERISTICS OF EVEN DIMENSIONAL TRIANGULATED MANIFOLDS
}

\author{
TOSHIYUKI AKITA \\ (Communicated by Alexander N. Dranishnikov)
}

\begin{abstract}
An alternative formula for the Euler characteristics of even dimensional triangulated manifolds is deduced from the generalized Dehn-Sommerville equations.
\end{abstract}

A finite simplicial complex $K$ is called an Eulerian manifold (or a semi-Eulerian complex in the literature) if all of the maximal faces have the same dimension and, for every nonempty face $\sigma \in K$,

$$
\chi(\operatorname{Lk} \sigma)=\chi\left(S^{\operatorname{dim} K-\operatorname{dim} \sigma-1}\right)
$$

holds, where $\operatorname{Lk} \sigma$ is the link of $\sigma$ in $K$ and $S^{n}$ is the $n$-dimensional sphere. Note that $K$ is not necessarily connected. Any triangulation of a closed manifold is an Eulerian manifold. More generally, a triangulation of a homology manifold without boundary provides an Eulerian manifold. The purpose of this short note is to prove the following alternative formula for the Euler characteristics of even dimensional Eulerian manifolds.

Theorem 1. Let $K$ be a $2 m$-dimensional Eulerian manifold. Then

$$
\chi(K)=\sum_{i=0}^{2 m}\left(-\frac{1}{2}\right)^{i} f_{i}(K)
$$

holds, where $f_{i}(K)$ is the number of $i$-simplices of $K$.

A finite simplicial complex $L$ is called a flag complex if every collection of vertices of $L$ which are pairwise adjacent spans a simplex of $L$. The formula (1) was proved in [1] under the additional assumptions that $K$ is a PL-triangulation of a closed $2 m$-manifold and is a flag complex. M. W. Davis pointed out that the formula (1) follows from a result in [3, provided $K$ is a flag complex (see Note added in proof in (1). Both results follow from the considerations of the Euler characteristics of Coxeter groups. In this note, we deduce formula (1) from the generalized DehnSommerville equations proved by Klee [4].

Received by the editors January 31, 2007.

2000 Mathematics Subject Classification. Primary 52B70; Secondary 52B05, 57Q15.

The author was partially supported by the Grant-in-Aid for Scientific Research (C) (No. 17560054) from the Japan Society for Promotion of Sciences.

(C)2008 American Mathematical Society Reverts to public domain 28 years from publication 
Let $K$ be a finite $(d-1)$-dimensional simplicial complex and $f_{i}=f_{i}(K)$ the number of $i$-simplices of $K$ as before. The $d$-tuple $\left(f_{0}, f_{1}, \ldots, f_{d-1}\right)$ is called the $f$-vector of $K$. The $f$-polynomial $f_{K}(t)$ of $K$ is defined by

$$
f_{K}(t)=t^{d}+f_{0} t^{d-1}+\cdots+f_{d-2} t+f_{d-1} .
$$

Define the $h$-polynomial $h_{K}(t)$ of $K$,

$$
h_{K}(t)=h_{0} t^{d}+h_{1} t^{d-1}+\cdots+h_{d-1} t+h_{d},
$$

by the rule $h_{K}(t)=f_{K}(t-1)$. The $(d+1)$-tuple $\left(h_{0}, h_{1}, \ldots, h_{d}\right)$ is called the $h$-vector of $K$. The $h$-vector of $K$ satisfies the generalized Dehn-Sommerville equations, as stated below in Theorem 2 ,

Theorem $2(4])$. Let $K$ be $a(d-1)$-dimensional Eulerian manifold. Then

$$
h_{d-i}-h_{i}=(-1)^{i}\left(\begin{array}{l}
d \\
i
\end{array}\right)\left(\chi(K)-\chi\left(S^{d-1}\right)\right)
$$

holds for all $i(0 \leq i \leq d)$.

Remark. Klee stated the generalized Dehn-Sommerville equations in terms of the $f$ vector rather than the $h$-vector. The formulae quoted in Theorem 2 are equivalent to those in [4] and can be found in [5]. Theorem 2 was also proved in [2] by a quite different method, provided that $K$ is a triangulation of a closed manifold.

Now we prove Theorem 1, We have

$$
h_{K}(-1)=\sum_{i=0}^{2 m+1}(-1)^{2 m+1-i} h_{i}=\sum_{i=0}^{m}(-1)^{i}\left(h_{2 m+1-i}-h_{i}\right) .
$$

Now Theorem 2 asserts that

$$
h_{2 m+1-i}-h_{i}=(-1)^{i}\left(\begin{array}{c}
2 m+1 \\
i
\end{array}\right)(\chi(K)-2) .
$$

Hence we obtain

$$
h_{K}(-1)=(\chi(K)-2) \sum_{i=0}^{m}\left(\begin{array}{c}
2 m+1 \\
i
\end{array}\right)=2^{2 m}(\chi(K)-2) .
$$

On the other hand, we have

$$
f_{K}(-2)=(-2)^{2 m+1}+\sum_{i=0}^{2 m}(-2)^{2 m-i} f_{i}=2^{2 m}\left(-2+\sum_{i=0}^{2 m}\left(-\frac{1}{2}\right)^{i} f_{i}\right) .
$$

Since $h_{K}(-1)=f_{K}(-2)$ by the definition of the $h$-polynomial $h_{K}(t)$, Theorem 1 follows from (2) and (3).

\section{REFERENCES}

1. T. Akita, Euler characteristics of Coxeter groups, PL-triangulations of closed manifolds, and cohomology of subgroups of Artin groups, J. London Math. Soc. (2) 61 (2000), 721-736. MR 1766100(2001f:20080)

2. V. M. Buchstaber, T. E. Panov, Torus actions and their applications in topology and combinatorics, University Lecture Series 24, American Mathematical Society, Providence, RI, 2002. MR:1897064(2003e:57039)

3. R. Charney, M. W. Davis, Reciprocity of growth functions of Coxeter groups, Geom. Dedicata 39 (1991), 373-378. MR1123152 (92h:20067) 
4. V. Klee, A combinatorial analogue of Poincaré's duality theorem, Canad. J. Math. 16 (1964), 517-531. MR0189039 (32:6466)

5. E. Swartz, From spheres to manifolds, preprint (2005).

Department of Mathematics, Hokkaido University, Sapporo, 060-0810 Japan

E-mail address: akita@math.sci.hokudai.ac.jp 